\title{
Crystal structure analysis reveals Pseudomonas PilY1 as an essential calcium-dependent regulator of bacterial surface motility
}

\author{
Jillian Orans ${ }^{a}$, Michael D. L. Johnson ${ }^{b, 1}$, Kimberly A. Coggan ${ }^{c, 1}$, Justin R. Sperlazza ${ }^{a}$, Ryan W. Heiniger, Matthew C. \\ Wolfgang ${ }^{c, d}$, and Matthew R. Redinbo ${ }^{a, b, 2}$
}

\begin{abstract}
${ }^{a}$ Department of Chemistry, University of North Carolina at Chapel Hill, CB 3290, Chapel Hill, NC 27599. ${ }^{b}$ Departments of Biochemistry and Biophysics, and 'Microbiology and Immunology, University of North Carolina at Chapel Hill, CB 7290, Chapel Hill, NC 27599. ${ }^{d}$ Cystic Fibrosis/Pulmonary Research and Treatment Center, University of North Carolina at Chapel Hill, CB 7248, Chapel Hill, NC, 27599.
\end{abstract}

Edited by John J. Mekalanos, Harvard Medical School, Boston, MA, and approved December 3, 2009 (received for review October 7, 2009)

\begin{abstract}
Several bacterial pathogens require the "twitching" motility produced by filamentous type IV pili (T4P) to establish and maintain human infections. Two cytoplasmic ATPases function as an oscillatory motor that powers twitching motility via cycles of pilus extension and retraction. The regulation of this motor, however, has remained a mystery. We present the $2.1 \AA$ A resolution crystal structure of the Pseudomonas aeruginosa pilus-biogenesis factor PilY1, and identify a single site on this protein required for bacterial translocation. The structure reveals a modified $\beta$-propeller fold and a distinct EF-hand-like calcium-binding site conserved in pathogens with retractile T4P. We show that preventing calcium binding by PilY1 using either an exogenous calcium chelator or mutation of a single residue disrupts Pseudomonas twitching motility by eliminating surface pili. In contrast, placing a lysine in this site to mimic the charge of a bound calcium interferes with motility in the opposite manner-by producing an abundance of nonfunctional surface pili. Our data indicate that calcium binding and release by the unique loop identified in the PilY1 crystal structure controls the opposing forces of pilus extension and retraction. Thus, PilY1 is an essential, calcium-dependent regulator of bacterial twitching motility.
\end{abstract}

calcium binding | human pathogen | microbiology | pilus biogenesis | structural biology

$T^{1}$ he pathogenesis of bacterial infections typically depends on microbial adherence to host tissue, aggregation of the pathogen at the site of infection, and subsequent dissemination to other anatomical sites within the host. Type IV pili (T4P) are thin surface filaments involved in biofilm formation, bacterial aggregation, and microbial adherence to biotic and abiotic surfaces. $\mathrm{T} 4 \mathrm{P}$ biogenesis requires more than a dozen proteins conserved across a range of bacterial species (1). A subset of bacteria, including the opportunistic human pathogen Pseudomonas aeruginosa, cyclically extend and retract their T4P, facilitating a form of surface movement termed twitching motility (2-4). Retractile T4P are required for $P$. aeruginosa adherence to host tissue, virulence, and dissemination of infection (5-7).

The pilin subunits that form T4P are incapable of self-assembly. Instead, pilus extension and retraction requires repeated rounds of pilin polymerization and depolymerization, both of which demand ATP hydrolysis $(8,9)$. In P. aeruginosa, the cytoplasmic ATPase PilB drives the assembly of pilin monomers into mature T4P $(8,10)$, while the structurally related cytoplasmic ATPase PilT disassembles T4P back into pilin monomers $(8,11,12)$. Loss-of-function mutations in PilT result in the loss of twitching motility due to the inability of formed pilus fibers to retract (12). The C-terminal half of the $117-\mathrm{kDa}$ P. aeruginosa PilY1 protein shares sequence homology with the C-terminal domain (CTD) of the PilC proteins of pathogenic Neisseria species; in contrast, the N-terminal regions of the PilCs and PilY1 are divergent in sequence (13). Although the PilC proteins from
Neisseria have been implicated in antagonizing pilus retraction by the pilus-biogenesis ATPase PilT, the N-terminal domains of these proteins appear critical for host cell attachment (14, 15). Thus, we hypothesized that the conserved CTDs of PilY1 and PilC may play a common role in T4P biogenesis rather than adhesion, and pursued a structural and functional analysis of the CTD from Pseudomonas aeruginosa PilY1.

\section{Results}

PilY1 Exhibits a Modified $\beta$-Propeller Fold. Examination of the amino acid sequence of PilY1 from P. aeruginosa revealed conservation between its C-terminal 550 residues and the same regions of the PilC proteins from Neisseria, and a predicted structural homology to the eight-bladed $\beta$-propeller fold exhibited by a range of proteins $(16,17)$. Thus, we overexpressed, purified, and crystallized the PilY1 CTD (amino acids 6145-1163) for examination by x-ray diffraction. Native diffraction data were collected to $2.1 \AA$ A resolution (Table 1); however, attempts to determine the structure using existing $\beta$-propeller search models or by isomorphous replacement methods were not successful. PilY1 CTD contains only six methionine residues, which upon selenomethionine substitution failed to produce an adequate signal to support structure determination by anomalous dispersion (18). To overcome this problem, a series of single leucine-to-methionine (L-M) substitutions were placed in the PilY1 CTD to provide additional sites for single-wavelength anomalous dispersion (SAD) phasing (Figs. S1 and S2). After several attempts, three L-M substitutions (at positions $712,812,823$ ) were successfully combined, crystallized, and generated clear selenomethionine fluorescence signal upon examination in the $\mathrm{x}$-ray beam. The structure was determined by SAD phasing using crystals containing nine total selenomethionine residues per protein monomer (Table 1).

The structured 505 residues of the PilY1 CTD exhibit a sevenbladed modified $\beta$-propeller fold composed of $31 \beta$-strands and 9 $\alpha$-helices (Fig. $1 A$ and Figs. S1 and S2; residues at the termini, as well as 713-721 and 1061-1068 were disordered and are not in the refined model). Blades I through IV are four-stranded antiparallel $\beta$-sheets and align well with blades of a canonical $\beta$-propeller enzyme, quinohemoprotein alcohol dehydrogenase from Comamonas testosteroni [Protein Data Bank (PDB)

Author contributions: J.O., M.D.J., K.A.C., M.C.W., and M.R.R. designed research; J.O., M.D.J., K.A.C., J.R.S., and R.W.H. performed research; J.O., M.D.J., K.A.C., J.R.S., R.W.H., M.C.W., and M.R.R. analyzed data; and J.O., M.D.J., K.A.C., M.C.W., and M.R.R. wrote the paper. The authors declare no conflict of interest.

This article is a PNAS Direct Submission.

Data deposition: The atomic coordinates and structure factors have been deposited in the Protein Data Bank, www.pdb.org (PDB ID code 3HX6).

${ }^{1}$ M.D.L.J. and K.A.C. contributed equally to this work

${ }^{2}$ To whom correspondence should be addressed. E-mail: redinbo@unc.edu.

This article contains supporting information online at www.pnas.org/cgi/content/full/ 0911616107/DCSupplemental. 
Table 1. Crystallographic data collection, phasing, and refinement

PilY1 C-terminal domain

\begin{tabular}{|c|c|c|}
\hline Data set & Native & SeMet \\
\hline Unit cell, $a b c, \AA ; \alpha \beta \gamma,{ }^{\circ}$ & $a=64.4, b=108.3, c=159.0$ & $a=169.8, b=64.9, c=116.6, \beta=108.7$ \\
\hline Space group & $\mathrm{P} 22_{1} 2_{1} 2_{1}$ & $\mathrm{C} 2$ \\
\hline Wavelength, $\AA$ & 1.0718 & 0.9792 \\
\hline X-ray source & APS SER-CAT 22-BM & APS SBC-CAT 19-ID \\
\hline Resolution, ̊̊ (highest shell) & $50-2.10(2.18-2.10)$ & $50-2.80(2.90-2.80)$ \\
\hline No. of reflections, unique & 65477 & 29869 \\
\hline Completeness, \% & $99.7(98.8)$ & $99.9(100.0)$ \\
\hline$R_{\text {sym }} *, \%$ & $8.5(43.5)$ & $7.1(24.4)$ \\
\hline$I / \sigma$ & $39.7(6.7)$ & $48.6(9.8)$ \\
\hline Redundancy & $\begin{array}{l}13.9(13.3) \\
\text { SAD phasing statistics to } 2.8 \AA\end{array}$ & $7.8(7.9)$ \\
\hline Se atom sites in $\mathrm{AU}$ (observed/total) & & $14 / 18$ \\
\hline \multicolumn{3}{|l|}{ Mean figure of merit } \\
\hline MLPHARE (highest shell) & & $0.65(0.60)$ \\
\hline After DM (highest shell) & & $0.82(0.41)$ \\
\hline & Refinement summary & \\
\hline Resolution, Å (highest shell) & $50-2.10(2.18-2.10)$ & \\
\hline Molecules per AU & 2 & \\
\hline No. of protein atoms/AU & 7,361 & \\
\hline No. of waters/AU & 818 & \\
\hline$R_{\text {working }}{ }^{\dagger}, \%$ & $19.6(23.7)$ & \\
\hline$R_{\text {free }}{ }^{\ddagger} \% \%$ & $23.5(28.1)$ & \\
\hline \multicolumn{3}{|l|}{ Average B factor, $\AA^{2}$} \\
\hline Protein & 29.5 & \\
\hline Water & 42.3 & \\
\hline \multicolumn{3}{|l|}{ rms deviations } \\
\hline Bonds, $\AA$ & 0.005 & \\
\hline Angles, ${ }^{\circ}$ & 1.37 & \\
\hline \multicolumn{3}{|l|}{ Coordinate error estimates, $\AA$} \\
\hline Luzzati & 0.23 & \\
\hline SigmaA & 0.15 & \\
\hline \multicolumn{3}{|l|}{ Ramachandran, \% } \\
\hline Allowed, generous, disallowed & $98.6,1.0,0.4$ & \\
\hline Protein Data Bank ID code & $3 \mathrm{H} \times 6$ & \\
\hline
\end{tabular}

AU, asymmetric unit; APS, Advanced Photon Source; SER, Southeast Regional; CAT, Collaborative Access Team; SBC, Structural Biology Center; BM, bending magnet; ID, insertion device; SAD, single-wavelength anomalous dispersion; MLPHARE, maximum likelihood heavy atom refinement and phase calculation; DM, density modification.

${ }^{*} R_{\text {sym }}=\Sigma|I-\langle I\rangle| / \Sigma|I|$, where $I$ is the observed intensity and $\langle I\rangle$ is the average intensity of several symmetry-related observations.

${ }^{\dagger} R_{\text {working }}=\Sigma|| F_{o}|-| F_{c}|| / \Sigma\left|F_{o}\right|$, where $F_{o}$ and $F_{c}$ are the observed and calculated structure factors, respectively.

${ }^{\ddagger} R_{\text {free }}=\Sigma|| F_{o}|-| F_{c}|| / \Sigma\left|F_{o}\right|$ for $5 \%$ of the data not used at any stage of the structural refinement.

1KB0; overall rmsd of 3.6 Å over 290 equivalent $\mathrm{C} \alpha$ positions with $15 \%$ sequence identity; Fig. $1 B]$ (16). This $\beta$-propeller enzyme was identified by Phyre to align with the PilY1 predicted structure ( $E$-value $0.014,95 \%$ certainty) (16). Blades V through VII of the PilY1 CTD, however, deviate from the standard $\beta$-propeller fold and align poorly with established $\beta$-propellers. Blade $\mathrm{V}$ contains a fifth strand $(\beta 20)$ that is shared with blade VI. Blade VI is composed of six strands, including $\beta 20$, and maintains an insert containing a short helix and $\beta$-turn- $\beta$ motif $(\alpha 9, \beta 26-27)$. Finally, blade VII is truncated and composed of three relatively short strands.

WD40 $\beta$-propeller proteins mediate protein-protein interactions via surface contacts at the propeller center (19). Superposition of the PilY1 CTD structure onto that of the seven-bladed $\beta$-propeller WDR5, complexed with a lysine 4-containing histone H3 peptide, revealed an rmsd of $3.9 \AA$ over 261 equivalent $\mathrm{C} \alpha$ positions sharing only $12 \%$ sequence identity (20). Blades I-IV of the PilY1 CTD align well with the first four blades of WDR5, whereas the last three blades of each protein deviate more significantly in structure (Fig. S3). However, helices four and seven of PilY1 appear to block the surface site used by WDR5 to interact with peptide (19). Both helices are predicted to be conserved in the C-terminal domains of the related Neisseria PilC proteins (Fig. S2) (17). These observations suggest that PilY1 and related pilus-biogenesis factors do not mediate pro- tein-protein interactions using the canonical WD40 surfacebinding site.

PilY1 Contains a Unique Calcium-Binding Loop. A strong $\left|F_{o}\right|-\left|F_{c}\right|$ electron density peak in the PilY1 CTD structure was interpreted as a calcium ion based on the observed coordination by three aspartic acid side chains $(851,855,859)$, one asparagine side chain (853), one main-chain carbonyl oxygen of valine 857 , and one water (Fig. $2 A$ and Fig. S1). The calcium binding observed in PilY1 is similar to that seen in the canonical EF-hand Ca-binding motifs (Fig. 2B and Fig. S4) (21). PilY1 and EF hands both employ seven contacts to the bound calcium ion, including one from a water molecule and one bidendate contact via an acidic side chain. PilY1 is unique, however, in that it achieves this calcium chelation using a stretch of only nine amino acids (851-859) in a loop between two $\beta$-strands ( $\beta 13$ and $\beta 14)$ rather than the twelve residues between two $\alpha$-helices typically observed in the EF hands (21). In addition, the carboxylic acid group of the bidendate Asp859 residue is rotated nearly $90^{\circ}$ with respect to the equivalent residue in EF-hand proteins (21). Asp-859 forms a hydrogen bond with His-797, which is conserved in PilY1 proteins of known sequence. The calcium-chelating residues observed in the structure are conserved in the PilY1s of known sequence and in the related PilCs, with the exception that Asn- 853 is replaced with an aspartic acid in the Neisseria proteins (Fig. $2 C$ and Fig. S2). This 


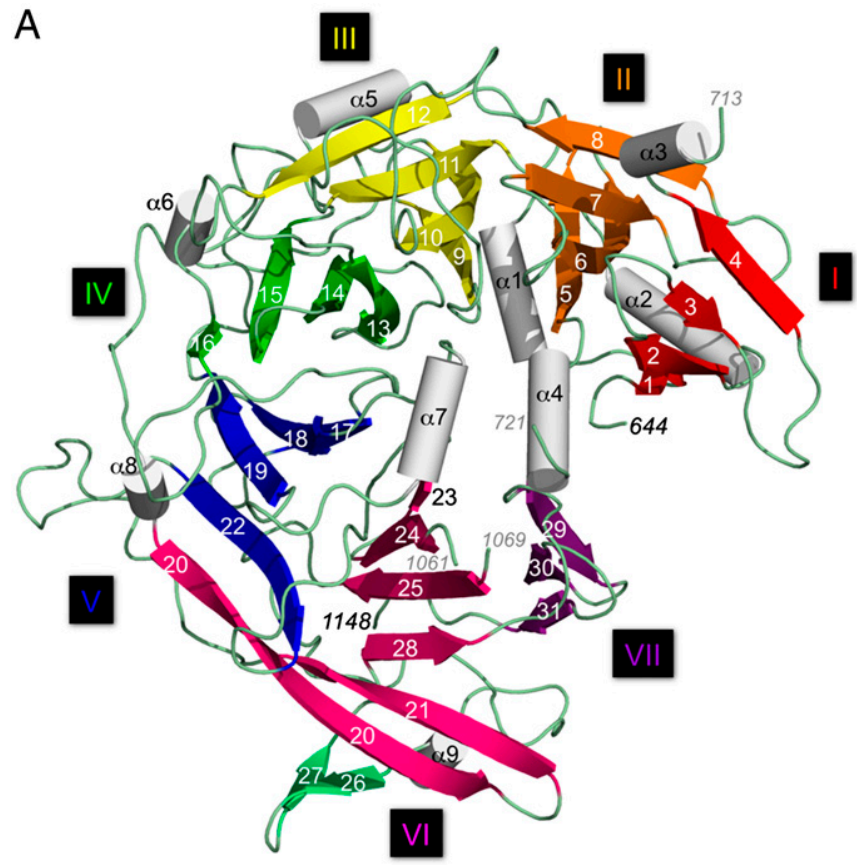

B

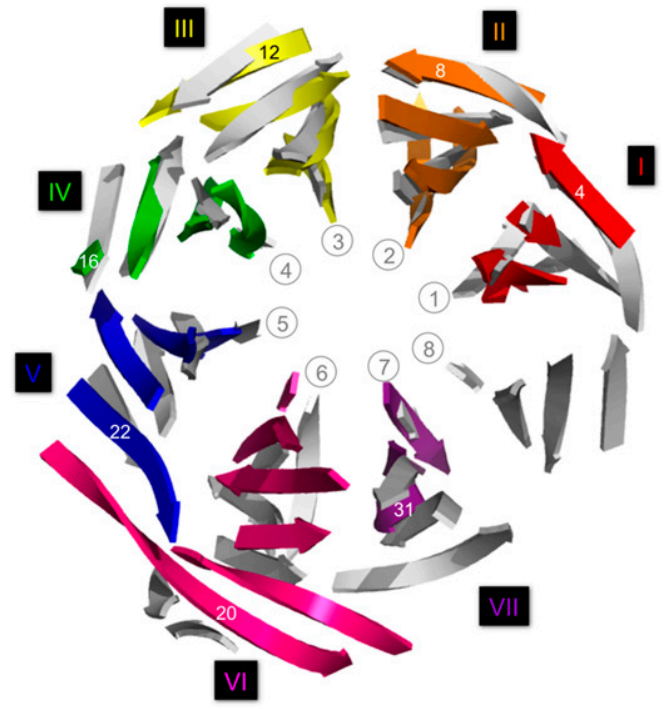

Fig. 1. Crystal structure of the P. aeruginosa PilY1 CTD. (A) $2.1 \AA \AA$ resolution crystal structure of the $P$. aeruginosa PilY1 C-terminal domain, which begins at residue 644 and ends at 1148 and encompasses seven $\beta$-blades (I-VII; colored red, orange, yellow, green, blue, magenta, and violet). (B) Superposition of the seven-bladed PilY1 CTD $\beta$-propeller (red through violet) on the eight-bladed $\beta$-propeller of the quinohemoprotein alcohol dehydrogenase from Comamonas testosteroni (1KB0; gray).

modified EF-hand-like motif is also present in PilY1 orthologues found in other bacteria that utilize T4P, including the human pathogen Kingella kingae. Thus, a unique calcium-binding site appears to be conserved in the C-terminal domains of pilusbiogenesis proteins related to PilY1.

Measurement of calcium-binding affinity using a fluorescence competition assay established that purified PilY1 CTD exhibits a $K_{d}$ of $2.6 \mu \mathrm{M}$ for calcium (Fig. $2 D$ ), which is similar to the affinities reported for traditional EF-hand proteins (22). Titration with increasing concentrations of magnesium chloride reveals that this cation does not bind to PilY1. Mutation of the calcium-chelating residue Asp-859 to alanine eliminates specific calcium binding by the protein (Fig. $2 D$ ). Removal of calcium either by Chelex 100 or by mutating a chelating residue (e.g.,
Fig. $2 D$ and Fig. S5) did not change the overall structure (as measured by circular dichroism spectropolarimetry; Fig. S6) or melting temperature (Fig. S7) of the PilY1 CTD (23). Thus, calcium binding appears not to play a role in the overall structure of the PilY1 C-terminal domain.

Calcium Binding and Release by PilY1 are Essential for Functional Pili. Expression of mutant versions of PilY1 in P. aeruginosa revealed that calcium binding and release are essential for pilus production and twitching motility. To examine the phenotypic consequences of altering the PilY1 calcium-binding site on T4P production and function, we engineered plasmid-encoded versions of full-length wild-type PilY1 and PilY1 carrying the D859A substitution. Both proteins were expressed to native levels in a $P$. aeruginosa strain in which the chromosomal pilY1 gene was deleted (Fig. S8). The PilY1 ${ }^{\text {minus }}$ strain is devoid of surface T4P, lacks measurable twitching motility, and is phenotypically indistinguishable from a nonpiliated PilA ${ }^{\text {minus }}$ strain, which does not produce the major T4P subunit, pilin (Fig. $3 A$ and $B$ ). Complementation of the PilY1 ${ }^{\text {minus }}$ strain with plasmid-expressed PilY1 restored twitching motility and pilus production to wild-type levels (Fig. $3 A$ and $B$ ). In contrast, complementation of the PilY $1^{\text {minus }}$ strain with full-length PilY1 D859A resulted in significantly less twitching motility $(p<0.001)$ (Fig. $3 A)$. The level of surface T4P produced by the PilY1 D859A expressing strain was also dramatically reduced compared to wild type and likely accounts for the observed defect in twitching motility (Fig. 3B).

PilY1 was previously shown to traffic to the surface T4P fraction (13) (Fig. S8), where it presumably functions through a direct association with the pilus fiber. To determine whether the defect in twitching observed with the D859A form of PilY1 was caused by disrupted pilus assembly/extension or increased pilus retraction, the PilY1 D859A mutant was expressed in a mutant strain lacking the PilT-retraction ATPase (PilT ${ }^{\text {minus }}$, PilY1 $1^{\text {minus }}$ strain). These bacteria regained the ability to produce normal levels of T4P, and the mutant PilY1 protein was capable of trafficking to the sheared pilus fraction (Fig. $3 C-F$ ). This result indicates that the loss of surface pili observed with the PilY1 D859A mutant is caused by PilT-mediated pilus retraction. In the presence of PilT, the D859A form of PilY1 is incapable of antagonizing PilT-mediated pilus retraction despite normal trafficking of the protein. We conclude that the calcium-binding site in the PilY1 CTD is necessary for normal pilus function by antagonizing the retractile activity of PilT.

We then created a mutation in full-length PilY1 designed to mimic calcium binding and to potentially render the protein calcium insensitive. Specifically, a lysine residue was substituted for the bidentate calcium-chelating Asp-859 residue (D859K). Although a lysine residue is not equivalent to a divalent metal ion, modeling of the D859K substitution mutation indicates that the $\varepsilon$-amino group of lysine would be expected to form a salt bridge with the two proximal aspartic acids at 851 and 855 (Fig. S9). Purified D859K PilY1 CTD does not bind calcium and is not distinct in structure or melting temperature from the wild-type CTD (Figs. S5-S7). When expressed to native levels in the $P$. aeruginosa PilY1 ${ }^{\text {minus }}$ strain (Fig. S8), the full-length PilY1 D859K mutant was significantly reduced for bacterial twitching motility $(p<0.001)$ compared to the same strain expressing wild-type PilY1 (Fig. 3A). Interestingly, the level of surface pilus production in the PilY1 D859K expressing strain was equivalent to that of the wild-type strain (Fig. $3 B$ ), indicating that reduced fiber production does not account for the twitching motility defect. PilY1 D859K was capable of trafficking to the surface T4P fraction when expressed in both a pilY1 mutant background (Fig. S8) and a PilT ${ }^{\text {minus }}$, PilY1 ${ }^{\text {minus }}$ strain (Fig. $3 C-F$ ). Thus, using the crystal structure as a guide, we produced a single-site mutation that dramatically alters pilus function without affecting pilus production. Based on these data, we conclude that 
A

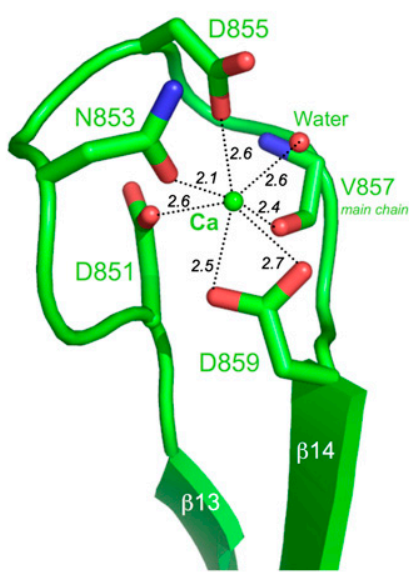

C

P.aer. PilY1

N.men. Pilc 1

N.men. Pilc2

N.gon. Pilc1

N.gon. Pilc2
851-DNNSDGVAD-859

728-DKDLDGTVD-7 36

738-DKDLDGTVD-746

736-DKDLDGTAD- 744

741-DKDLDGTVD- 749
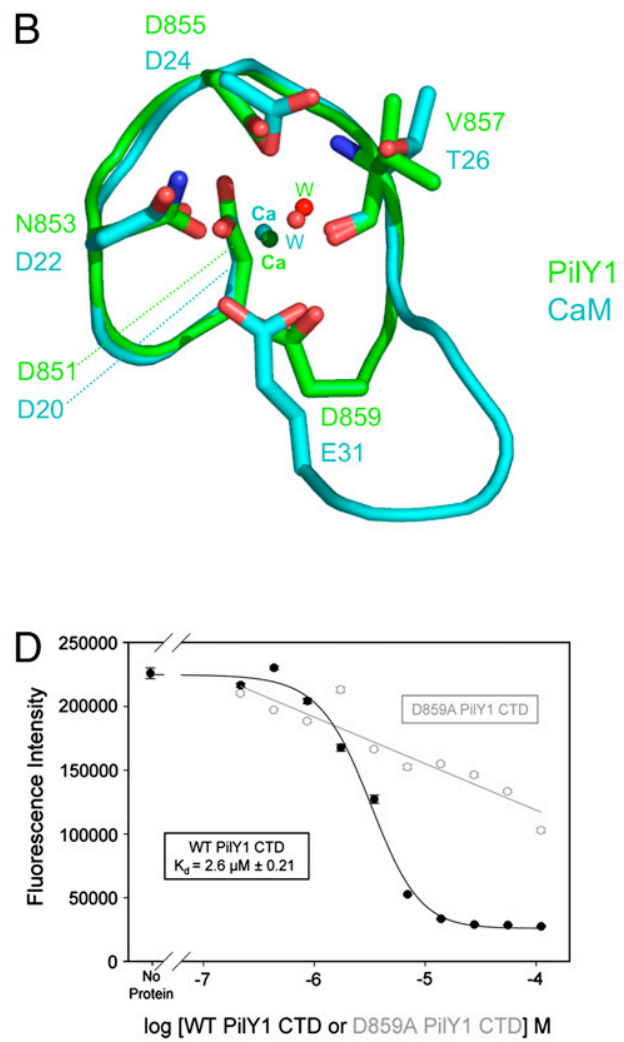

Fig. 2. PilY1 calcium-binding site. (A) The PilY1 calcium-binding site was revealed in clear electron density to be composed of aspartic acids 851,855 , and 859 , as well as asparagine 853 , the main-chain carbonyl oxygen of valine 857 , and a water molecule (red sphere). Distances are in angstroms. (B) Superposition of the nine-residue calcium-binding site of PilY1 (green) on the cannonical 12-residue site in human calmodulin (CaM; cyan). The calcium atoms (Ca) and water molecules (W) are depicted as spheres. Viewed in roughly the same orientation as in Fig. $2 A$. (C) Sequence conservation in the calcium-binding sites of the PilY1 homologues PilC1 and PilC2 from Neisseria meningitidis (N.men.) and N. gonorrhoeae (N.gon.). (D) The PilY1 CTD binds to calcium with a $K_{d}$ of $2.6 \mu \mathrm{M}$ (black), while the Asp-859-Ala mutant form of the CTD exhibits only nonspecific calcium binding (gray). Error represents SD.

the release of calcium by PilY1 is required for PilT-dependent fiber retraction and twitching motility.

Finally, we tested whether the calcium chelator EGTA impacts $P$. aeruginosa pilus production. We found that EGTA reduces the levels of surface pili present on bacteria expressing wild-type PilY1, whereas the nonretractile PilT ${ }^{\text {minus }}$ strain is blind to calcium chelation (Fig. 4A). Similarly, the D859K form of PilY1 is insensitive to the effects of EGTA and supports the production of wild-type levels of surface pili (Fig. $4 A$ ). These data show that the PilY1 D859K mutant, mimicking the calcium-bound state of PilY1, has increased capacity to antagonize PilT-mediated pilus retraction. Taken together, these cell-based studies support the conclusion that both calcium binding and calcium release by the unique site in PilY1 are essential for the proper regulation of pilus retraction dynamics and twitching motility.

\section{Discussion}

Based on the structural and functional data presented here, we propose that the PilY1 C-terminal domain exists in two critical states: calcium-bound, which inhibits PilT-mediated pilus retraction, and calcium-free, which does not inhibit PilT and allows pilus retraction to proceed. We further propose that the interconversion between these two states in wild-type PilY1 is required for the cycles of pilus extension and retraction necessary for twitching motility (Fig. 4B, Top). The D859A mutant form of PilY1 appears to mimic the calcium-free state of the protein and is not capable of antagonizing PilT-mediated retraction; thus, the equilibrium is shifted toward retraction, similar to that seen with the PilY1 1 minus strain (Fig. 4B). Calcium chelation by EGTA also shifts the equilibrium toward retraction. In contrast, the D859K variant mimics the calcium-bound state, shifting the equilibrium toward pilus extension and producing an effect similar to that seen with the PilT ${ }^{\text {minus }}$ strain (Fig. $4 B$ ). The relatively moderate $2 \mu \mathrm{M}$ calcium-binding affinity exhibited by PilY1 is in the range where cycles of calcium binding and release would be expected to be physiologically relevant. Thus, we conclude that PilY1 acts as a calcium-dependent regulator of the opposing forces responsible for T4P-mediated twitching motility. However, we stress that this is a functional hypothesis; the physical contacts between PilY1 and other factors necessary to achieve this regulation have yet to be elucidated. It is interesting to note that calcium binding by bacterial pseudopilins has recently been shown to be critical for functional type II secretion, a process believed to be analogous to T4P biogenesis and function (24). Thus, calcium may play an important role in several aspects of pilus biogenesis and regulation.

Twitching motility mediated by PilY1's influence on the PilB/ PilT oscillatory motor would be expected to be coordinated with the significant $>100 \mathrm{pN}$ retractile motion exerted by the system once target cell adhesion occurs $(25,26)$. Surface translocation by twitching motility likely involves relatively moderate affinity contacts between a surface and T4P (26). For example, a disulfide loop in pilin, the major pilin subunit of the pilus fiber, has been proposed to be a point of contact between surfaces and T4P (27). We propose that the cycles of pilus extension and retraction actuated by PilY1's C-terminal domain may control twitching motility involving these moderate affinity contacts. Once target host cell contact has been achieved, however, the T4P system would be expected to convert to a rapidly retracting, PilT-dominated state in which nearly nanonewton forces are exerted $(28,29)$. The function of this mode would be to quickly draw the bacterial cell into close contact with host tissue cells. It is tempting to speculate that 

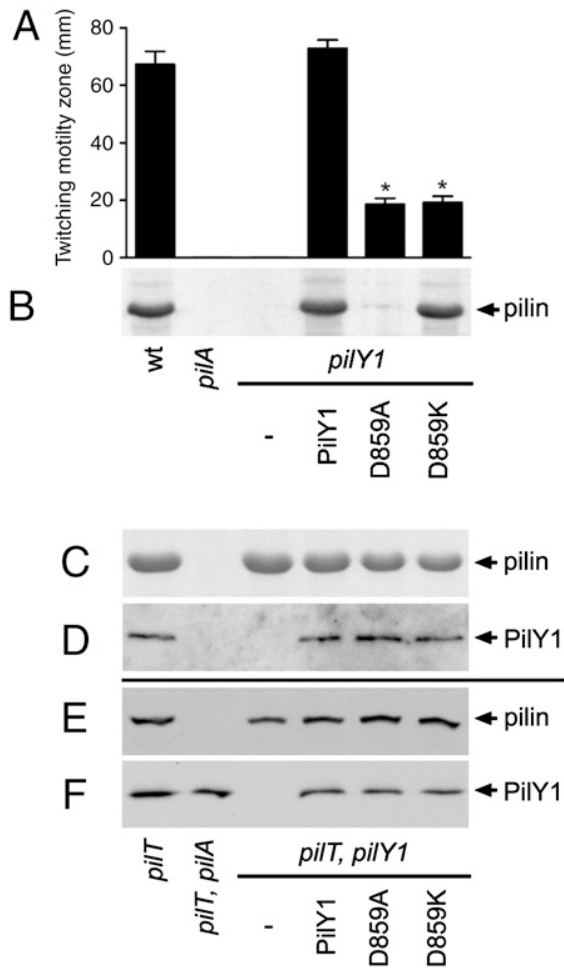

Fig. 3. PilY1 calcium-binding site is essential for T4P production and function. (A) Twitching motility of $P$. aeruginosa (WT) or a pilY1 mutant expressing either empty vector (-), PilY1, or mutant versions of PilY1 (D859A or D859K). The twitching motility zones produced by bacteria expressing mutant PilY1 were significantly $(*, p<0.001)$ reduced compared to those of bacteria expressing wild-type PilY1. (B) Coomassie blue-stained gel of pilin isolated from the bacterial surface. $(C-F)$ Abolishing T4P retraction allows conditional localization of PilY1 to the bacterial surface. (C) Coomassiestained gel of pilin isolated from the bacterial surface. $(D)$ Western blot of T4P-containing surface fractions probed with PilY1-specific antiserum. $(E)$ Western blot of whole cell lysates probed with a pilin-specific antibody (F) Western blot of whole cell lysates probed with a PilY1-specific antiserum.

PilY1 may serve as the switch between twitching and rapid retraction. The sequence divergent $\mathrm{N}$-terminal domains of PilY1 orthologues are proposed to perform target cell-specific adhesion; once that is achieved, perhaps the conserved C-terminal
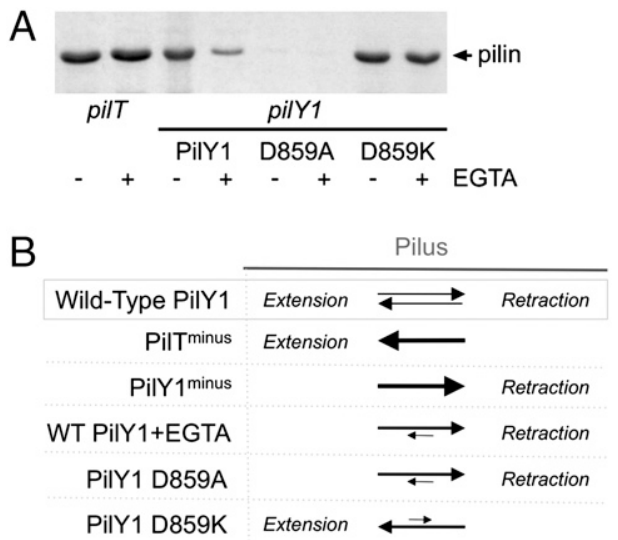

Fig. 4. Calcium chelation impacts T4P production. (A) Pilus preparations from a pilT mutant or a pilY1 mutant expressing either PilY1, or mutant versions of PilY1 grown in the presence $(+)$ or absence $(-)$ of the calcium chelator EGTA. (B) Summary of the impact specific mutants have on pilus state. Wildtype PilY1 appears to balance extension and retraction to produce twitching motility (boxed), whereas gene deletions, PilY1 site mutants, and the calcium chelator EGTA all significantly disrupt the equilibrium between pilus extenstion and retraction. domains switch to the calcium-free state to facilitate rapid PilT-mediated retraction $(14,30,31)$.

The C-terminal domain of $P$. aeruginosa PilY1 shares significant sequence identity with the equivalent regions of the Neisseria PilC's (Fig. 2C) and similar levels of identity in PilY1 homologues from other human, plant, and marine infectious bacteria (17). We find that each PilY1 homologue contains a predicted calciumbinding site highly similar in sequence to that observed in the PilY1 C-terminal domain (Fig. S2). Thus, calcium binding and release may play an equally important role in pilus biogenesis and motility in organisms that maintain a PilY1 homologue. Taken together, the structural and functional data we outline provide unique insights into the complex functional regulation of the T4P essential to a range of human pathogenic bacteria.

\section{Methods}

Structure Determination. PilY1 residues 615-1163 were cloned from genomic DNA from the PAK strain of $P$. aeruginosa (GenBank \#EU234515), and overexpressed recombinantly in $E$. coli. The protein was purified using $\mathrm{Ni}$ affinity and size-exclusion chromatography steps to $>95 \%$ purity, and crystallized in $0.3 \mathrm{M}$ sodium malonate ( $\mathrm{pH} 6.5$ ), 20\% (wt/vol) PEG 3350, $50 \mathrm{mM}$ DTT, $30 \mathrm{mM}$ ammonium phosphate, and 4-8\% trifluoroethanol. Residues 6145-1163 of PilY1 contained only five methionine residues; as such, crystals containing selenomethionine-substituted protein failed to produce sufficient signal to allow structure determination. L712M, L812M, and L823M mutations were successfully generated, combined, expressed, purified, and crystallized in PilY1 specimen sufficient for $x$-ray data collected. Diffraction data to $2.8 \AA$ resolution were collected at the peak wavelength (0.979 $\AA$ ) for selenium SAD phasing (Table 1 ) and indexed and scaled in space group C2 using $\operatorname{HKL} 3000(32,33)$. Automated model building correctly built $30 \%$ of the final structure; complete building and refinement was accomplished using the programs COOT and CNS, respectively $(34,35)$. Once the selenomethionine-substituted structure was refined at $2.8 \AA$ resolution, it was used in molecular replacement to solve the $2.1 \AA$ native structure, which was refined using COOT and CNS to the final statistics shown in Table $1(34,35)$.

Calcium Binding and Biophysical Studies. Calcium was removed from purified PilY1 using Chelex 100 (BioRad) prior to biophysical studies (23). A binding curve for Oregon Green ${ }^{\circledR} 488$ BAPTA-5N (Invitrogen) was measured on a PHERAstar (BMGLabtech) at $488 \mathrm{~nm}$ and the $K_{d}$ established at $10.1 \mu \mathrm{M}$. WT, D859A, and D859K PilY1 CTD was serial diluted 2-fold to calculate PilY1 CTD $K_{d}$ for calcium binding, if applicable. For biophysical characterizations via circular dichroism spectropolarimetry, a wavelength scan from 195-260 was performed at $22^{\circ} \mathrm{C}$. Thermal denaturation protein samples were measured $210 \mathrm{~nm}$ at a range of $10-85^{\circ} \mathrm{C}$.

Isolation of Surface T4P. $P$. aeruginosa strains were spread on LB agar plates and grown until confluent. Bacteria were collected and suspended in $1 \mathrm{ml}$ $0.15 \mathrm{M} \mathrm{NaCl} / 0.2 \%$ formaldehyde and vortexed vigorously for $1 \mathrm{~min}$ to release surface T4P. Bacterial cells were removed by centrifugation at $12,000 \times g$ for $5 \mathrm{~min}$. T4P fractions were separated by SDS-PAGE ( $18 \%$ polyacrylamide) and pilin visualized by GelCode blue stain (Pierce).

Twitching Motility. Bacteria were stab inoculated to the bottom of $100 \mathrm{~mm}$ tissue culture-treated plates containing $5 \mathrm{~mL}$ LB agar ( $1 \% \mathrm{wt} / \mathrm{vol})$. Plates were incubated for $40 \mathrm{~h}$ at $37^{\circ} \mathrm{C}$ in a humidified incubator. Twitching motility was quantified by measuring the diameter of the subsurface zone of bacterial spread.

Western Blots. Whole cell lysates and T4P fractions were separated on $18 \%$ (pilin) or $7.5 \%$ (PilY1) SDS-polyacrylamide gels and transferred to nitrocellulose or PVDF membranes, respectively. Membranes were probed with PKL1 antipilin monoclonal antibody or anti-PilY1 CTD rabbit serum. Horseradish peroxidase-conjugated secondary antibodies were used for the detection of specific antibody-antigen complexes. Blots were developed with chemiluminescence reagents and visualized via autoradiography.

ACKNOWLEDGMENTS. We thank Wladik Minor and Zbyszek Otwinowski for HKL3000 guidance, Joseph Lomino and Lisa Charlton of the Redinbo Laboratory for help with CD experiments, and Rebekah Nash and members of the Redinbo and Wolfgang groups for assistance with manuscript preparation. Supported by National Institutes of Health Grants Al078924 (to M.R.R.) and Al069116 (to M.C.W.), and a Howard Hughes Medical Institute Medinto-Grad Initiative Training Grant (K.A.C.). 
1. Craig L, Li J (2008) Type IV pili: Paradoxes in form and function. Curr Opin Struct Biol, 18:267-277.

2. Merz AJ, So M, Sheetz MP (2000) Pilus retraction powers bacterial twitching motility. Nature, 407:98-102.

3. Skerker JM, Berg HC (2001) Direct observation of extension and retraction of type IV pili. Proc Natl Acad Sci USA, 98:6901-6904.

4. Wolfgang M, et al. (1998) PilT mutations lead to simultaneous defects in competence for natural transformation and twitching motility in piliated Neisseria gonorrhoeae. Mol Microbiol, 29:321-330.

5. Comolli JC, et al. (1999) Pseudomonas aeruginosa gene products PilT and PilU are required for cytotoxicity in vitro and virulence in a mouse model of acute pneumonia. Infect Immun, 67:3625-3630.

6. Mattick JS (2002) Type IV pili and twitching motility. Annu Rev Microbiol, 56:289-314

7. Tang H, Kays M, Prince A (1995) Role of Pseudomonas aeruginosa pili in acute pulmonary infection. Infect Immun, 63:1278-1285.

8. Chiang $P$, et al. (2008) Functional role of conserved residues in the characteristic secretion NTPase motifs of the Pseudomonas aeruginosa type IV pilus motor proteins PilB, PilT and PilU. Microbiology, 154:114-126.

9. Jakovljevic V, Leonardy S, Hoppert M, Sogaard-Andersen L (2008) PilB and PilT are ATPases acting antagonistically in type IV pilus function in Myxococcus xanthus. J Bacteriol, 190:2411-2421.

10. Turner LR, Lara JC, Nunn DN, Lory S (1993) Mutations in the consensus ATP-binding sites of $\mathrm{XcpR}$ and PilB eliminate extracellular protein secretion and pilus biogenesis in Pseudomonas aeruginosa. J Bacteriol, 175:4962-4969.

11. Morand PC, et al. (2004) Type IV pilus retraction in pathogenic Neisseria is regulated by the PilC proteins. Embo J, 23:2009-2017.

12. Whitchurch $\mathrm{CB}$, et al. (1991) Characterisation of a Pseudomonas aeruginosa twitching motility gene and evidence for a specialised protein export system widespread in eubacteria. Gene, 101:33-44.

13. Alm RA, Hallinan JP, Watson AA, Mattick JS (1996) Fimbrial biogenesis genes of Pseudomonas aeruginosa: pilW and pilX increase the similarity of type 4 fimbriae to the GSP protein-secretion systems and pilY1 encodes a gonococcal PilC homologue. Mol Microbiol, 22:161-173.

14. Rudel T, Scheurerpflug I, Meyer TF (1995) Neisseria PilC protein identified as type-4 pilus tip-located adhesin. Nature, 373:357-359.

15. Wolfgang $M$, et al. (1998) Suppression of an absolute defect in type IV pilus biogenesis by loss-of-function mutations in pilT, a twitching motility gene in Neisseria gonorrhoeae. Proc Natl Acad Sci USA, 95:14973-14978.

16. Kelley LA, Sternberg MJ (2009) Protein structure prediction on the Web: A case study using the Phyre server. Nat Protoc, 4:363-371.

17. Subramaniam S (1998) The biology workbench-A seamless database and analysis environment for the biologist. Proteins, 32:1-2.
18. Hendrickson WA, Horton JR, LeMaster DM (1990) Selenomethionyl proteins produced for analysis by multiwavelength anomalous diffraction (MAD): A vehicle for direct determination of three-dimensional structure. Embo J, 9:1665-1672.

19. Smith TF, Gaitatzes C, Saxena K, Neer EJ (1999) The WD repeat: A common architecture for diverse functions. Trends Biochem Sci, 24:181-185.

20. Holm L, Kaariainen S, Rosenstrom P, Schenkel A (2008) Searching protein structure databases with DaliLite v.3. Bioinformatics, 24:2780-2781

21. Chattopadhyaya R, Meador WE, Means AR, Quiocho FA (1992) Calmodulin structure refined at $1.7 \mathrm{~A}$ resolution. $J \mathrm{Mol} \mathrm{Biol}, 228: 1177-1192$

22. Weber C, Lee VD, Chazin WJ, Huang B (1994) High level expression in Escherichia coli and characterization of the EF-hand calcium-binding protein caltractin. J Biol Chem 269:15795-15802.

23. Suzuki H, Pabst MJ, Johnston RB Jr (1985) Enhancement by $\mathrm{Ca}^{2+}$ or $\mathrm{Mg}^{2+}$ of catalytic activity of the superoxide-producing NADPH oxidase in membrane fractions of human neutrophils and monocytes. J Biol Chem, 260:3635-3639.

24. Korotkov KV, et al. (2009) Calcium is essential for the major pseudopilin in the type 2 secretion system. J Biol Chem, 284:25466-25470.

25. Maier B, et al. (2002) Single pilus motor forces exceed 100 pN. Proc Natl Acad Sci USA 99:16012-16017.

26. Maier B, Koomey M, Sheetz MP (2004) A force-dependent switch reverses type IV pilus retraction. Proc Natl Acad Sci USA, 101:10961-10966.

27. Farinha MA, et al. (1994) Alteration of the pilin adhesin of Pseudomonas aeruginosa PAO results in normal pilus biogenesis but a loss of adherence to human pneumocyte cells and decreased virulence in mice. Infect Immun, 62:4118-4123.

28. Thomas W, et al. (2006) Catch-bond model derived from allostery explains forceactivated bacterial adhesion. Biophys J, 90:753-764.

29. Sokurenko EV, Vogel V, Thomas WE (2008) Catch-bond mechanism of force-enhanced adhesion: Counterintuitive, elusive, but... widespread?. Cell Host Microbe, 4:314-323.

30. Kirchner M, Heuer D, Meyer TF (2005) CD46-independent binding of neisserial type IV pili and the major pilus adhesin, PilC, to human epithelial cells. Infect Immun, 73:3072-3082.

31. Kallstrom H, Liszewski MK, Atkinson JP, Jonsson AB (1997) Membrane cofactor protein (MCP or CD46) is a cellular pilus receptor for pathogenic Neisseria. Mol Microbiol, 25:639-647

32. Otwinowski Z, Minor W (1997) Processing of x-ray diffraction data collected in oscillation mode. Methods Enzymol, 276:307-326.

33. Minor W, Cymborowski M, Otwinowski Z, Chruszcz M (2006) HKL-3000: The integration of data reduction and structure solution-from diffraction images to an initial model in minutes. Acta Crystallogr D, 62:859-866.

34. Cowtan PEaK (2004) Coot: Model-building tools for molecular graphics. Acta Crystal$\log D, 60: 2126-2132$.

35. Brunger AT, et al. (1998) Crystallography \& NMR system: A new software suite for macromolecular structure determination. Acta Crystallogr D, 54:905-921. 